\title{
Analysis of Ultrastructural Properties of Lung Type II Alveolar Epithelial Cells After Severe Acute Pancreatitis in Wild-type and Surfactant Protein D Knockout Mice
}

Han Chen ${ }^{1}$ and Guirong Wang ${ }^{2}$

${ }^{1}$ Penn State College of Medicine, Hershey, Pennsylvania, United States, ${ }^{2}$ SUNY Upstate Medical University, Syracuse, New York, United States

Severe acute pancreatitis (SAP) often causes significant multiple organ (i.e. lung and pancreas) injury by an inflammatory response with the involvement of a cascade of cellular and molecular events1. Surfactant protein D (SP-D), an innate immune molecule, plays critical roles in host defense and the regulation of inflammation 2. Our previous study has demonstrated that SP-D had a protective effect on the pancreatic injury in the cecal ligation and puncture (CLP) induced septic murine model3. The NLRP3 inflammasomes and nuclear factor kappa $\mathrm{B}(\mathrm{NF}-\kappa \mathrm{B})$ are key regulators of innate immunity and inflammatory signaling. We hypothesized that SP-D can attenuate inflammation and tissue injury by suppressing NLRP3 inflammasome activation and regulating NF- $\kappa$ B signaling pathway in experimental SAP. In order to reveal the cellular and molecular mechanisms in the pathogenesis of SAP and the SP-D effect we performed the detailed analyses of ultrastructural properties of lung type II alveolar epithelial cells using transmission electron microscopic technique in this specific project.

The results demonstrated that treatment with both caerulein and LPS caused multiple organ injury including lung and pancreas in all three types of (WT, KO and hTG) mice. SP-D KO mice showed more severe lung injury compared to WT mice $(\mathrm{P}<0.05)$. hTG mice exhibited similar lung injury as WT mice. The ultrastructural abnormalities of mitochondria are found in SP-D KO mice pulmonary endothelial cell. These abnormalities consist not only of a decrease of the number of mitochondria but also of an enlarged and abnormal shape, variations in the number of cristae and particular patterns of cristae, and abnormal inclusions. The functional consequences of these mitochondrial abnormalities can be far reaching and systemic due to the common underlying impairment of oxidative phosphorylation. In Fig 2, a detail from a pulmonary endothelial cell is shown with mitochondria that contain abnormal inclusions. EM analysis revealed that there are significant differences of autophagosome formation, mitochondrial and rough endoplasmic reticulum (rER) injury, and the number of ribosome bodies in the lung type II cells and pancreatic acinar cells between SAP WT and KO mice ( $\mathrm{p}<0.05)$. The levels of NLRP3 inflammasome (NLRP3, ASC and L-1 $\beta$ ) and NF-kB activation in the lungs of SAP KO mice were higher than that of SAP WT and hTG mice $(\mathrm{p}<0.05)$.

We conclude that SP-D plays a protective role of in the pathogenesis of SAP through regulating innate inflammation, autophagy, mitochondria dysfunction and apoptosis [4]. 


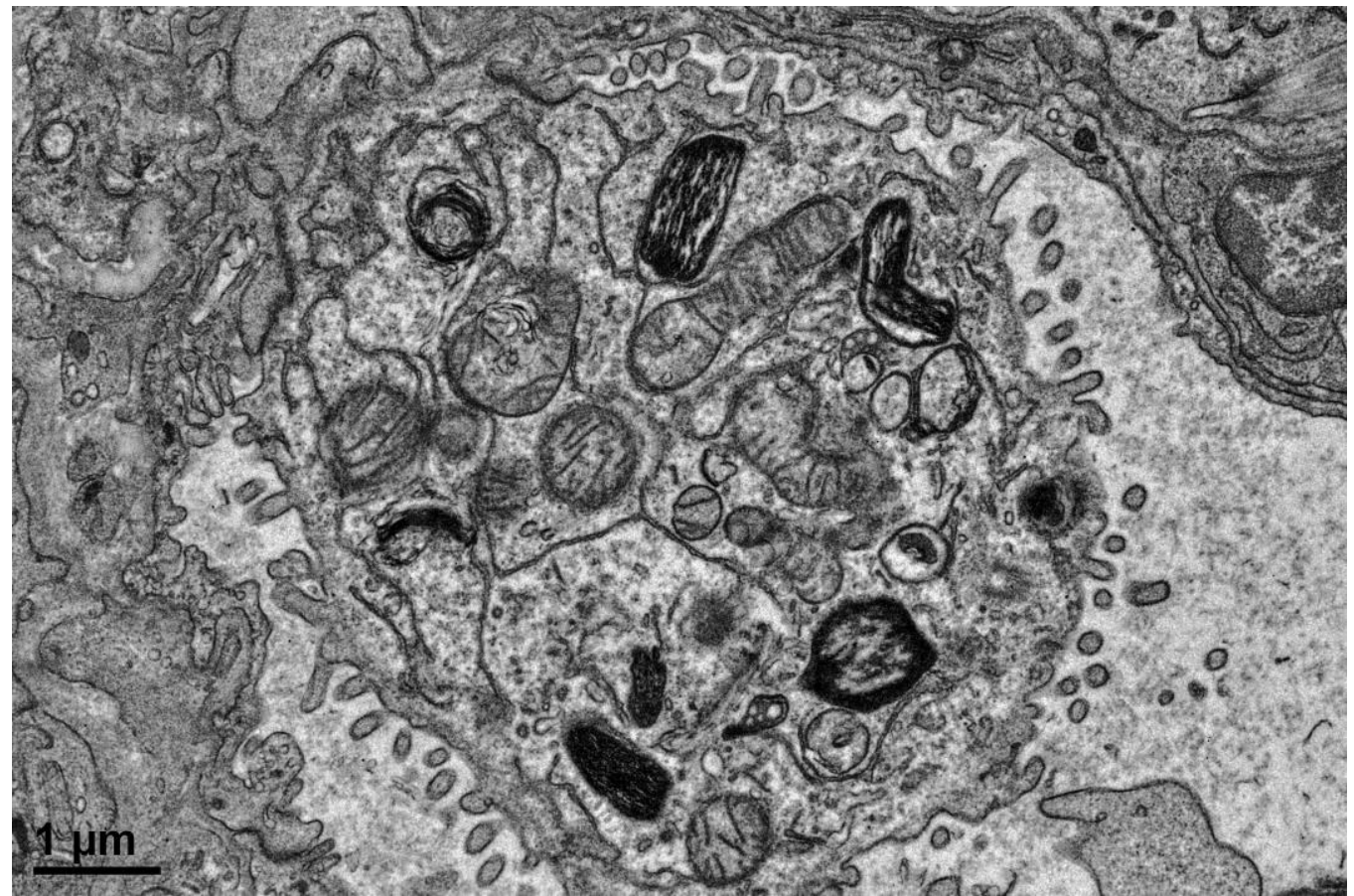

Figure 1. WT mice pulmonary endothelial cell. Scale bar 1um.

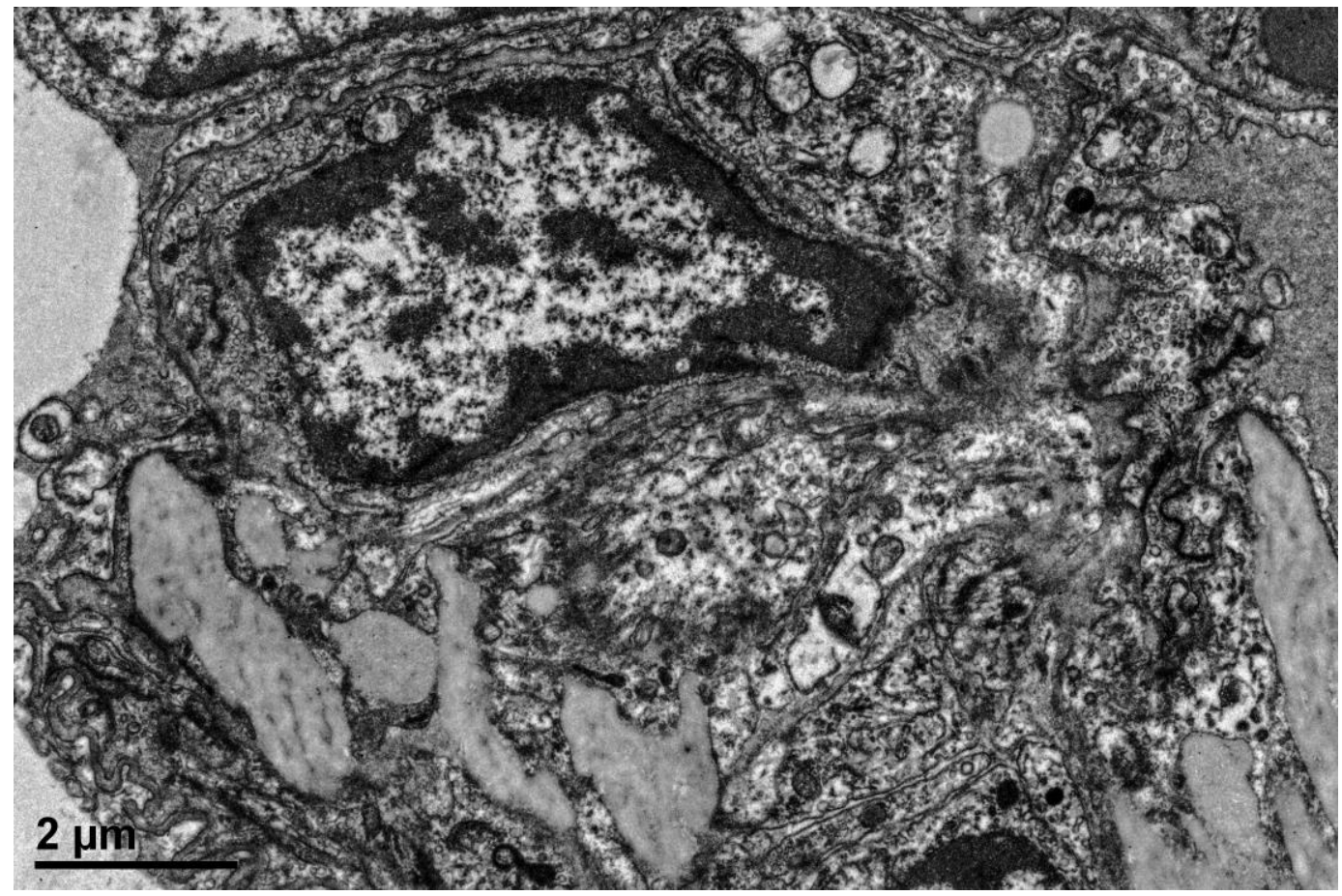

Figure 2. SP-D KO mice pulmonary endothelial cell. Scale bar 2 um.

\section{References}

[1] E Charbonney and AB Nathens, Surg Infect (Larchmt) 9 (2008), p.573.

[2] JR Wright. Nat Rev Immunol 5 (2005), p.58.

[3] Z Liu, et al. Sci Rep 5 (2015), p.17798.

[4] The authors acknowledge funding from NIH R01 HL136706 and NSF research award 172263. 